\title{
Été 2021 : des phénomènes météorologiques marquants dans le monde
}

\section{Michaël Kreitz' ${ }^{1}$ Matthieu Sorel $^{2}$}

1. École nationale de la météorologie, Météo-France

2. Direction de la climatologie et des services climatiques, Météo-France

ien que considéré comme maussade voire « pourri » sur la France (anomalie thermique de $+0,4{ }^{\circ} \mathrm{C}$ et anomalie pluviométrique de +23\%), l'été 2021 est marqué par de nombreux nouveaux records absolus de températures à travers le monde.

Sur notre territoire (se référer aux résumés climatiques dans ce numéro), le mois de juin connaît une forte anomalie chaude, alors que juillet et août sont en déficit de température. Toutefois, sur la saison complète de l'été météorologique, 2021 est plus chaud que la normale. Mais le climat évolue : il faut remonter à 2014 pour retrouver un été plus froid, les six derniers étés ayant été particulièrement chauds, voire exceptionnels. Ainsi, l'été 2021 se situe presqu'au niveau du fameux été 1976 (figure 1).

\section{Plusieurs records nationaux battus}

Côté froid, seulement trois records mensuels nationaux sont battus en juin, juillet et août et aucun record mensuel national absolu. Côté chaud, de très nombreux records mensuels nationaux sont battus sur la période estivale et plusieurs records nationaux absolus battus (tableau 1 et figure 2).

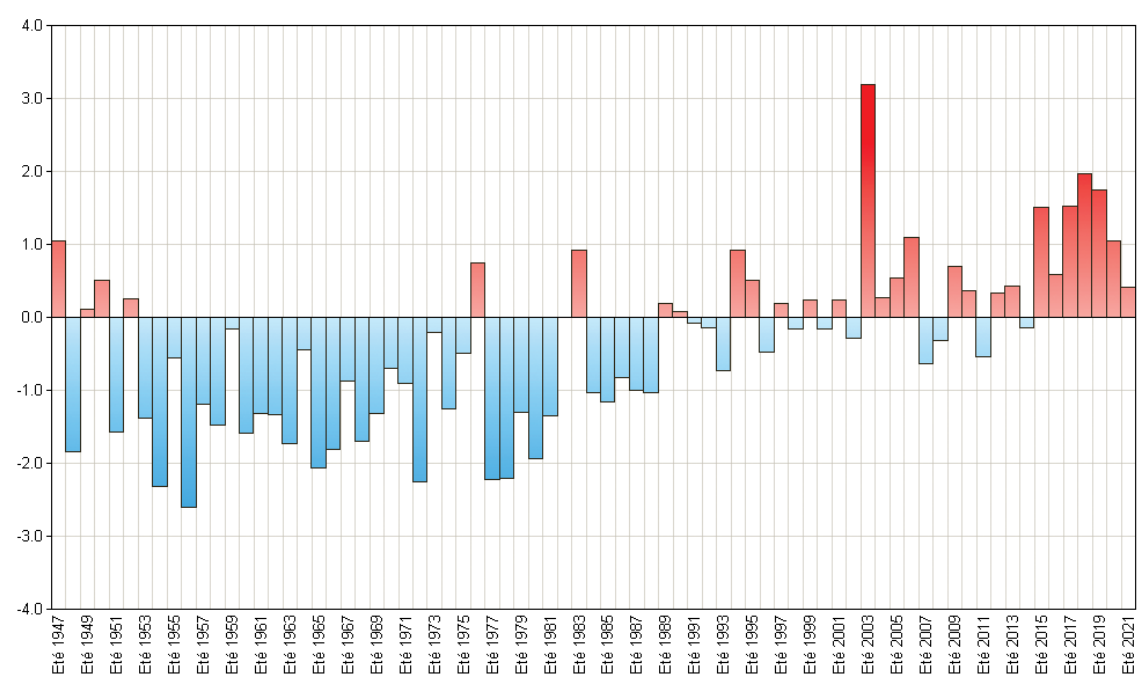

Figure 1. Anomalie de température moyenne estivale en France entre 1947 et 2021, la normale étant calculée sur la période 1981-2010. Source : Météo-France.

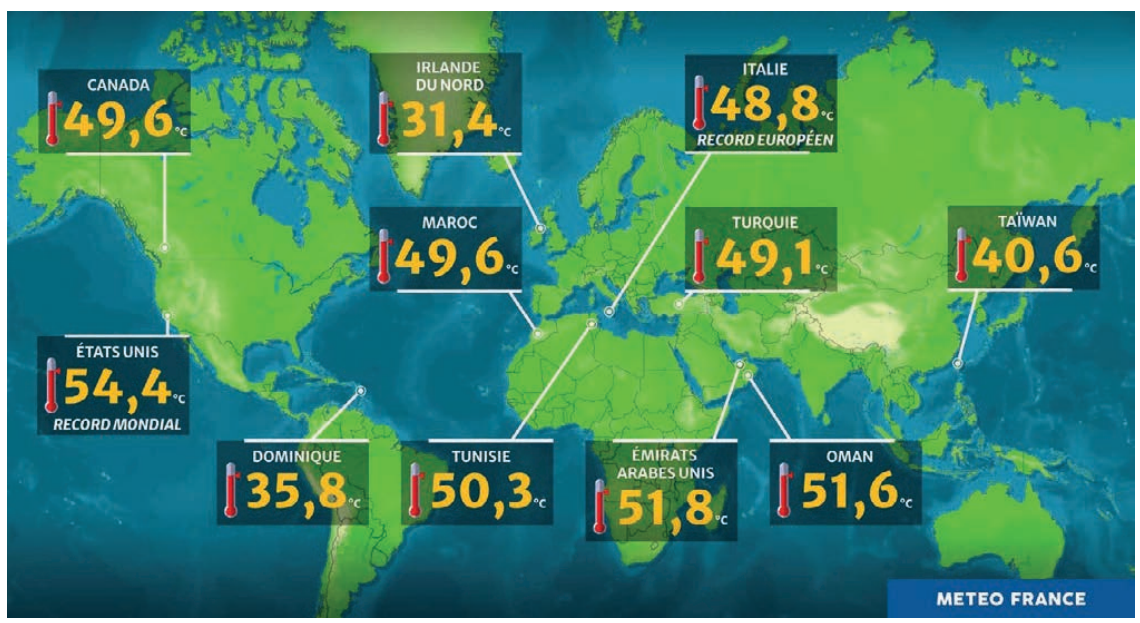

Figure 2. Principaux records nationaux de chaleur battus dans le monde. Source : Météo-France1. 
Tableau 1. Principaux records de températures battus durant l'été 2021. II s'agit de températures maximales absolues sauf pour quelques cas de températures minimales chaudes absolues signalées par Tn. Les records sont classés par ordre chronologique. Source : http://www.geoclimat.org/

\begin{tabular}{|c|c|c|c|c|}
\hline Pays & Station & Valeur & Date & Commentaire \\
\hline Émirats arabes unis & Sweihan & $51,8^{\circ} \mathrm{C}$ & 6 juin & \\
\hline Oman & Joba & $51,6^{\circ} \mathrm{C}$ & 16 juin & \\
\hline Canada & Lytton, BC & $49,6^{\circ} \mathrm{C}$ & 29 juin & Maximale absolue au nord de $45^{\circ} \mathrm{N}$ \\
\hline Canada & Vancouver, BC & $29,0^{\circ} \mathrm{C}(\mathrm{Tn})$ & 29 juin & $\begin{array}{l}\text { Plus haute température minimale jamais enregistrée au Canada. Valeur arrondie } \\
\text { à } 0,5^{\circ} \mathrm{C}\end{array}$ \\
\hline Tunisie & Tataouine & $35,5^{\circ} \mathrm{C}(\mathrm{Tn})$ & 30 juin & Plus haute température minimale jamais enregistrée en Tunisie \\
\hline $\begin{array}{l}\text { États-Unis } \\
\text { d'Amérique }\end{array}$ & $\begin{array}{l}\text { Furnace Creek, } \\
\text { CA }\end{array}$ & $\begin{array}{l}54,4^{\circ} \mathrm{C} \\
130^{\circ} \mathrm{F}\end{array}$ & 9 juillet & $\begin{array}{l}\text { Record mondial fiable, non officiel. Selon l'OMM² le record mondial est de } \\
56,7^{\circ} \mathrm{C} \text { le } 10 \text { juillet } 1913 \text {, valeur contestée par de nombreux climatologues. } \\
\text { Le } 16 \text { août } 2020,54,4^{\circ} \mathrm{C} \text { avaient déjà été relevés (Renaut, 2020) mais } \\
\text { il s'agissait de } 129,9^{\circ} \mathrm{F} \text { contre } 130,0^{\circ} \mathrm{F} \text { ici ! }\end{array}$ \\
\hline Maroc & Sidi Slimane & $49,6^{\circ} \mathrm{C}$ & 10 juillet & Record national fiable égalé \\
\hline $\begin{array}{l}\text { États-Unis } \\
\text { d'Amérique }\end{array}$ & Stovepipe Wells, CA & $42,1^{\circ} \mathrm{C}(\mathrm{Tn})$ & 12 juillet & $\begin{array}{l}\text { Plus haute température minimale jamais enregistrée aux États-Unis et en } \\
\text { Amérique du Nord. Record mensuel mondial. }\end{array}$ \\
\hline Turquie & Cizre & $49,1^{\circ} \mathrm{C}$ & 20 juillet & \\
\hline Irlande du Nord & Armagh & $31,4^{\circ} \mathrm{C}$ & 22 juillet & \\
\hline Taïwan & Taimalee & $40,6^{\circ} \mathrm{C}$ & 25 juillet & \\
\hline Tunisie & Kariouan & $50,3^{\circ} \mathrm{C}$ & 11 août & \\
\hline Italie & Syracuse, Sicile & $48,8^{\circ} \mathrm{C}$ & 11 août & Ancien record officiel de l'OMM³ de 48,0 ${ }^{\circ} \mathrm{C}$ à Athènes, Grèce le 10 juillet 1977. \\
\hline Dominique & Canefield & $35,8^{\circ} \mathrm{C}$ & 12 août & \\
\hline
\end{tabular}

2. https://wmo.asu.edu/content/world-highest-temperature

3. https://wmo.asu.edu/content/europe-highest-temperature

\section{Un dôme de chaleur exceptionnel au Canada}

L'ouest du Canada (Colombie Britannique) et le nord-ouest des États-Unis (Oregon, Washington) connaissent une vague de chaleur sans précédent en cette fin juin 2021. La mise en place de cet épisode est complexe et sa compréhension nécessite d'examiner la situation météorologique en amont de quelques jours. L'évacuation d'une masse d'air très humide en provenance d'Asie a renforcé le jet d'ouest du Pacifique Nord. Sur la figure 3, cela se traduit par un fort gradient de géopotentiel et d'iso-épaisseur le 23 juin au sud des îles Aléoutiennes. Ce jet engendre lui-même une cyclogenèse explosive au large de l'Amérique du Nord jusque sur l'Alaska, que l'on retrouve autour du 25 juin. En aval du flux, cette cyclogenèse engendre un déferlement anticyclonique d'ondes de Rossby prenant la forme d'un breaking event (figure 4). Concrètement, une dorsale de hautes valeurs (pression, géopotentiel, température) se met en place à tous les niveaux (on la devine dès le 25 juin sur les côtes ouest américaines et canadiennes) avant de se positionner sur l'ouest du Canada et des États-Unis. Le géopotentiel est extrêmement élevé en altitude, approchant même la valeur de 6000 mètres géopotentiels (mgp), ainsi que les iso-épaisseurs (valeurs supérieures à $5920 \mathrm{mgp}$ ) selon les analyses des 27 et 29 juin : un « dôme de chaleur » (encadré 1) se crée et persiste plusieurs jours sur cette région d'Amérique du Nord. La pression n'est pas si élevée en surface car l'effet thermique peut jouer (l'air chaud est plus léger et donc la pression réduite au niveau de la mer est moins élevée).

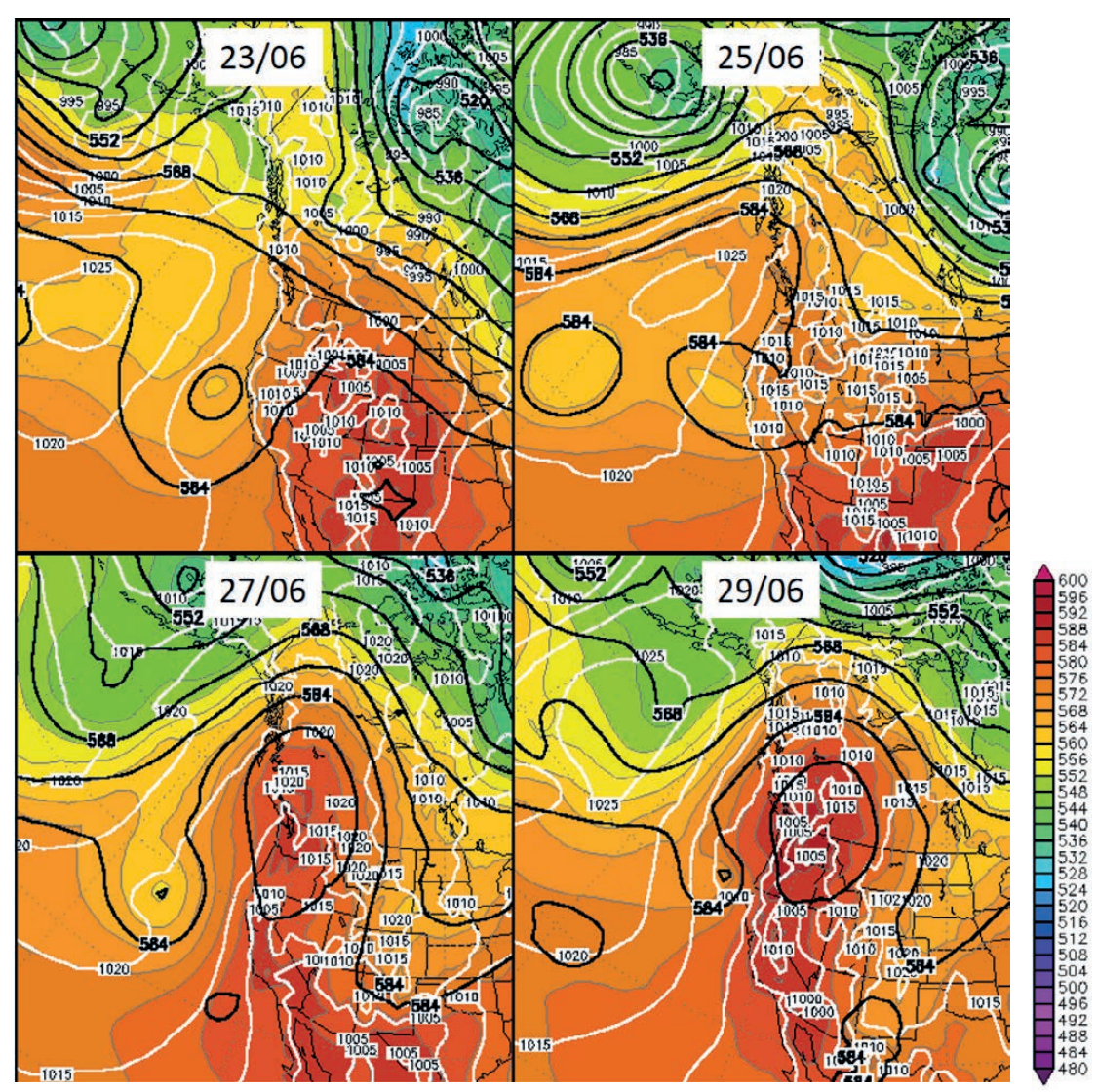

Figure 3. Pression réduite au niveau de la mer (Pmer, isolignes blanches), Géopotentiel au niveau $500 \mathrm{hPa}$ (plages de couleurs), épaisseur 500-1 $000 \mathrm{hPa}$ (décamètres géopotentiels, damgp, isolignes noires) analysées du 23 au 29 juin 2021 par le modèle américain GFS (Global Forecasting System) sur l'ouest de l'Amérique du Nord. Source : http://www1.wetter3.de/
Les rétro-trajectoires (figure 5a, Stein et al., 2015) confirment effectivement ces évolutions successives. Pour ce faire, des particules sont placées le 29 juin à $23 \mathrm{~h}$ TU sur la ville de Vernon (Colombie britannique) aux altitudes de 500, 1000 et 2000 mètres. 


\section{Dôme de chaleur}

Le terme " dôme de chaleur ", arrivé cet été dans les médias n'est pas une appellation météorologique, ni un phénomène nouveau. II s'agit d'une vaste zone anticyclonique (surtout en altitude) en période estivale persistant plusieurs jours. Concrètement, l'anticyclone est stable et très puissant. Les perturbations et le courant jet sont rejetés plus au nord du dôme formant une circulation de blocage, dite en oméga. Une puissante subsidence (réchauffement adiabatique par compression) a lieu, accentuant chaque jour la température.

En remontant le temps, leur origine est mise en évidence. Provenant du Pacifique Est où le courant perturbé sévissait quelques jours avant, elles franchissent ensuite les montagnes Rocheuses avec une évidente courbure anticyclonique. De plus, il ressort bien que ces particules proviennent

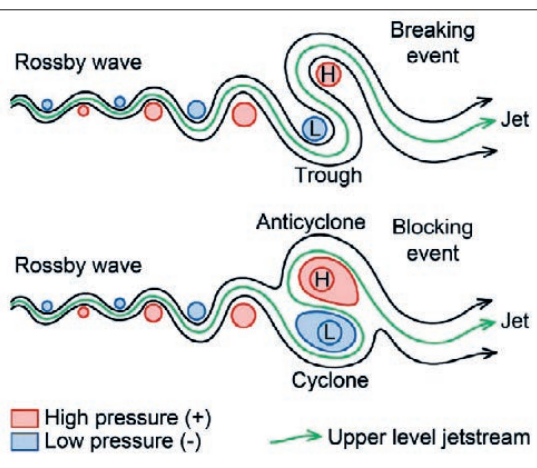

Figure 4. Schémas types de déferlement d'ondes de Rossby illustrant la distribution des hautes (high) et basses (low) pressions de part et d'autre du courant jet. Source : Mansouri Daneshvar et Freund, 2019. d'altitudes bien plus élevées (plus de 6000 mètres quelques jours avant). Elles subissent une subsidence progressive qui s'accentue durant les 48 dernières heures, au franchissement $\mathrm{du}$ relief. Il y a donc probablement une double cause à cette très nette et durable subsidence. Le radiosondage de Vernon (à quelques dizaines de kilomètres de Lytton seulement) illustre cette subsidence par une courbe d'état (courbe des températures) qui vient s'aligner sur une adiabatique sèche (courbes inclinées à environ $45^{\circ}$ ) jusqu'à 650 , voire même $500 \mathrm{hPa}$ (figure 5b) !

La présence de ce dôme de chaleur exceptionnel se traduit par de nombreux records absolus de températures au Canada et aux États-Unis. Le record national du Canada est battu plusieurs jours de suite pour finalement arriver à la valeur finale de $49,6{ }^{\circ} \mathrm{C}$ à la station de Lytton (figure 6) en Colombie Britannique (le précédent record absolu national était de $45,0{ }^{\circ} \mathrm{C}$ enregistré à Midale et Yellow Grass, État de Saskatchewan, en 1937). Cette valeur est saisissante de par la différence avec le précédent record et par sa proximité avec la barre symbolique des $50{ }^{\circ} \mathrm{C}$, alors que cette station est proche des $50^{\circ}$ $\mathrm{N}$ de latitude. D'ailleurs, cette valeur est un record pour l'ensemble des régions situées au nord du $45^{\mathrm{e}}$ parallèle. Pour replacer cette valeur dans son contexte, le record absolu de température au Canada est le même que celui du Maroc, lui aussi battu au cours de cet été 2021 . Le $1^{\text {er }}$ juillet, cette même ville de Lytton a été partiellement dévastée par un incendie. Ironie du

4. https://www.ready.noaa.gov/HYSPLIT.php 5. http://weather.uwyo.edu/upperair/sounding. html

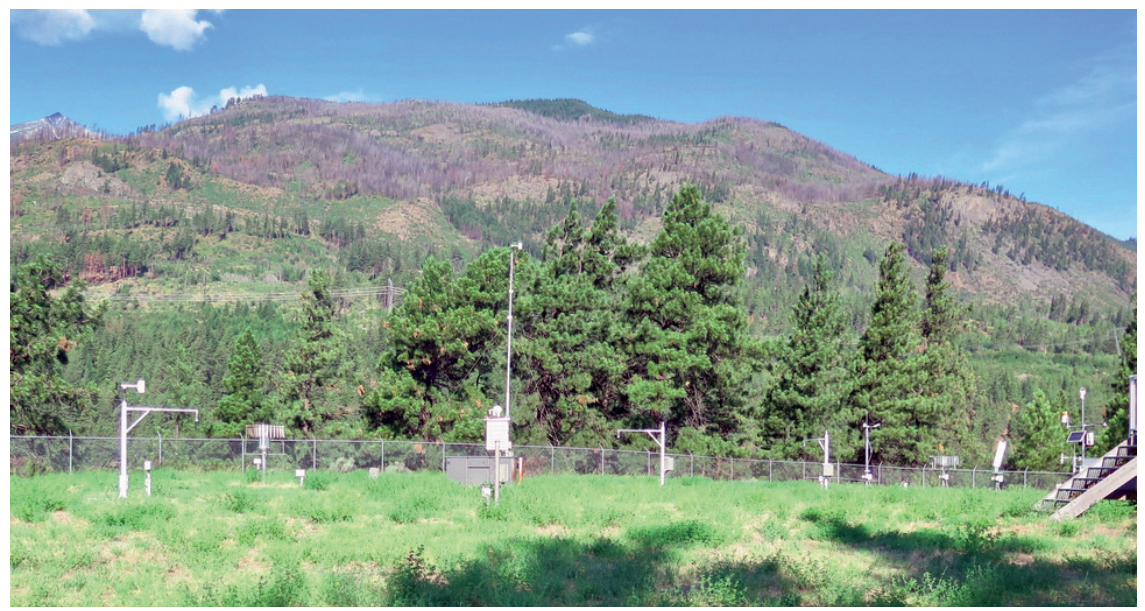

Figure 6. Station de Lytton en Colombie britannique. Source : Thierry Goose.
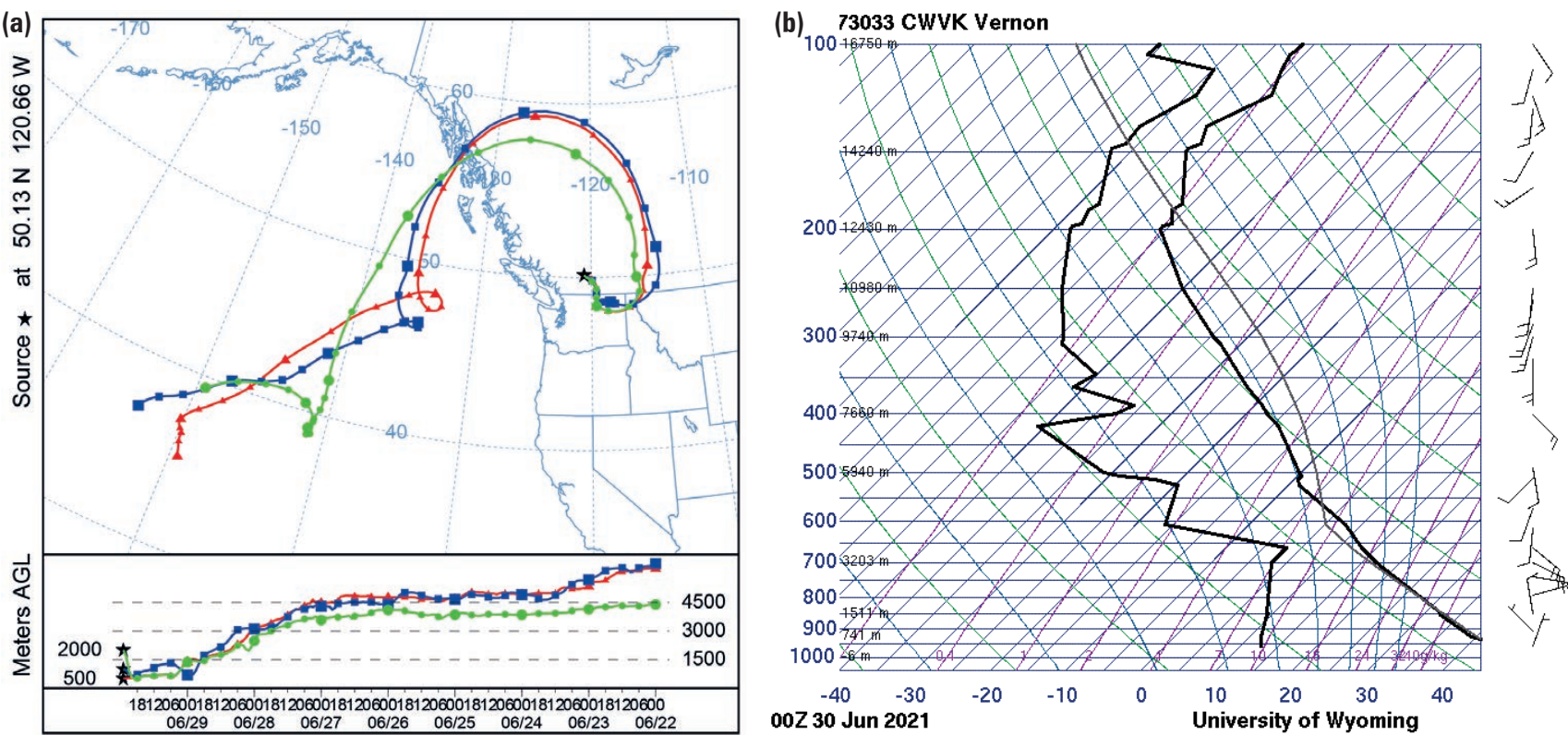

Figure 5. (a) rétrotrajectoires calculées à la verticale de Vernon, en partant d'une altitude de $500 \mathrm{~m}$ (rouge), $1000 \mathrm{~m}$ (bleu) et $2000 \mathrm{~m}$ (vert) le 29 juin 2021 à 23 h TU. Source : Noaa Air Resources Laboratory4. (b) Radiosondage réalisé à Vernon le 30 juin 2021 à 0 h TU. Source : université du Wyoming5. 
sort, la station météorologique enregistrant ce record a été épargnée par les flammes.

La nuit du 29 juin, la station Burnaby de l'université SFU dans l'agglomération de Vancouver enregistre une température minimale nocturne de $29,0{ }^{\circ} \mathrm{C}$ (valeur arrondie à $0,5{ }^{\circ} \mathrm{C}$ ), nouveau record absolu de température minimale pour le Canada. La station de Quillayute (État de Washington, États-Unis) pulvérise son record absolu de plus de $6,1^{\circ} \mathrm{C}$. Selon Maximiliano Herrera, un célèbre climatologue, il s'agit de la plus grosse différence au niveau mondial entre la première et la seconde plus haute valeur mesurée par une station détenant une longue période de données, ex aequo avec la station de Steel
Tableau 2. Inondations les plus coûteuses en Europe sur la période 1980-2020. Source : Munich Re.

\begin{tabular}{|c|c|c|c|c|c|}
\hline Date & Phénomènes & $\begin{array}{l}\text { Pays } \\
\text { concernés }\end{array}$ & $\begin{array}{l}\text { Pertes } \\
\text { (milliards } \\
\text { d'euros) }\end{array}$ & $\begin{array}{l}\text { Bien } \\
\text { assurés } \\
\text { (milliards } \\
\text { d'euros) }\end{array}$ & Victimes \\
\hline Août 2002 & $\begin{array}{l}\text { Inondations } \\
\text { et crues éclair }\end{array}$ & $\begin{array}{l}\text { Allemagne, Autriche, } \\
\text { République Tchèque, } \\
\text { Hongrie, Suisse, } \\
\text { Slovaquie }\end{array}$ & 22,496 & 4,916 & 39 \\
\hline 0ctobre 2000 & $\begin{array}{l}\text { Inondations et } \\
\text { glissements } \\
\text { de terrain }\end{array}$ & Italie et Suisse & 13,518 & 0,799 & 38 \\
\hline Novembre 1994 & $\begin{array}{l}\text { Inondations } \\
\text { et crues éclair }\end{array}$ & Italie & 12,591 & 0,088 & 68 \\
\hline $\begin{array}{l}\text { Juillet et } \\
\text { août } 1997\end{array}$ & Inondations & $\begin{array}{l}\text { Pologne, } \\
\text { République Tchèque, } \\
\text { Allemagne, Autriche } \\
\text { Slovaquie }\end{array}$ & 10,437 & 1,444 & 118 \\
\hline Mai et juin 2013 & Inondations & $\begin{array}{l}\text { Allemagne, Autriche, } \\
\text { République Tchèque, } \\
\text { Suisse, Hongrie }\end{array}$ & 10,219 & 2,424 & 25 \\
\hline
\end{tabular}

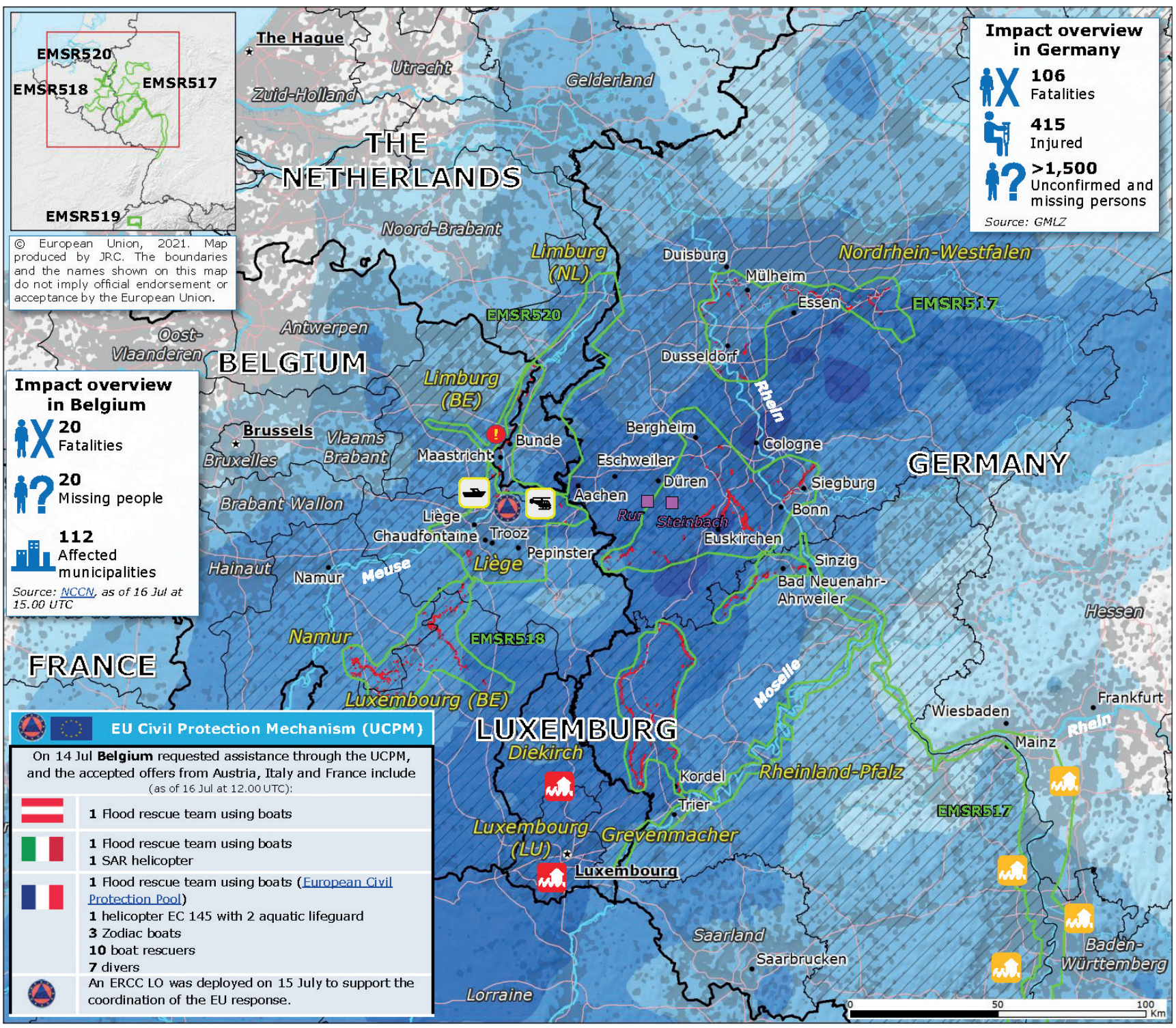

Figure 7. Régions d'Europe les plus concernées par les inondations du 14 juillet avec bilan provisoire en date du 16 juillet 2021 et esquisse des cumuls de précipitations sur trois jours. Source : JRC pour l'Union européenne. 

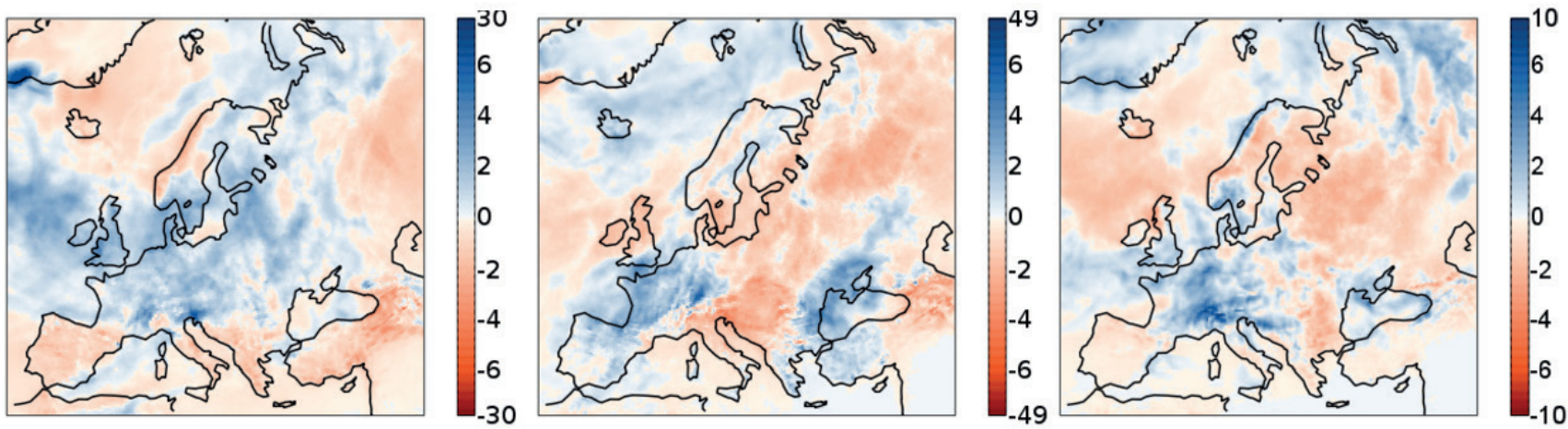

Figure 8. Anomalies de précipitations mensuelles (en mm/jour) de mai à juillet 2021. Source : https://climate.copernicus.eu/

(Dakota du Nord, États-Unis). La station de Montpellier-Fréjorgues avait battu son record absolu de $+5,8{ }^{\circ} \mathrm{C}$ lors de la canicule de juin 2019 et arrive pour l'instant au $4^{\mathrm{e}}$ rang mondial de ce classement déroutant. Les villes de Seattle (mesures depuis 1870) et de Portland (mesures depuis 1890) battent elles aussi leur record absolu avec respectivement $42,2{ }^{\circ} \mathrm{C}$ (station très proche de la mer) et $46,7^{\circ} \mathrm{C}$.

\section{Inondations en Allemagne autour de la mi-juillet}

Au-delà des valeurs remarquables de températures, l'été s'est accompagné d'épisodes pluvieux intenses, aboutissant à des inondations exceptionnelles. Les images venant d'Allemagne et du Benelux ont marqué les esprits. En effet, le 14 juillet, des pluies abondantes se déversent entre le nord-est de la France, l'Allemagne et le Benelux. Il est relevé pour ce jour-là jusqu'à 150, voire $200 \mathrm{~mm}$ en Allemagne, dans les régions de Rhénanie-du-NordWestphalie et Rhénanie-Palatinat au-

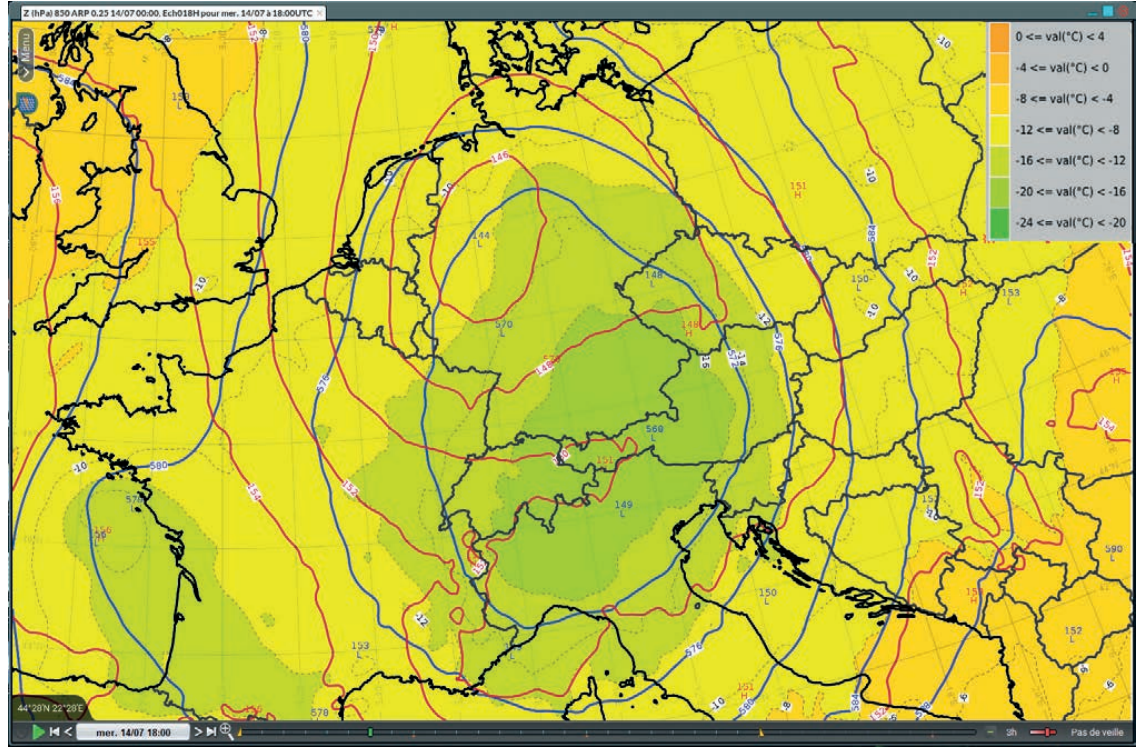

Figure 9. Géopotentiel (isolignes bleues en damgp), températures (couleurs) à $500 \mathrm{hPa}$ et Pmer (isolignes marrons) analysées par Arpège le 14 juillet 2021 à 18 h TU. Source : Météo-France.

tour de la ville de Cologne et, dès la veille, plus de $80 \mathrm{~mm}$ sont tombés localement. Les records de pluie en 24 heures sont pulvérisés. La figure 7 donne une représentation des régions les plus touchées, du bilan provisoire, ainsi que des moyens d'intervention mis à disposition par l'Union européenne en date du 16 juillet.
Le bilan définitif est extrêmement lourd avec près de 200 victimes en Allemagne et 40 en Belgique. Les dégâts sont difficilement chiffrables et aujourd'hui estimés à plus de 30 milliards d'euros. Selon la compagnie d'assurances Munich Re, il s'agirait des inondations les plus coûteuses en Europe depuis 1980 (tableau 2).
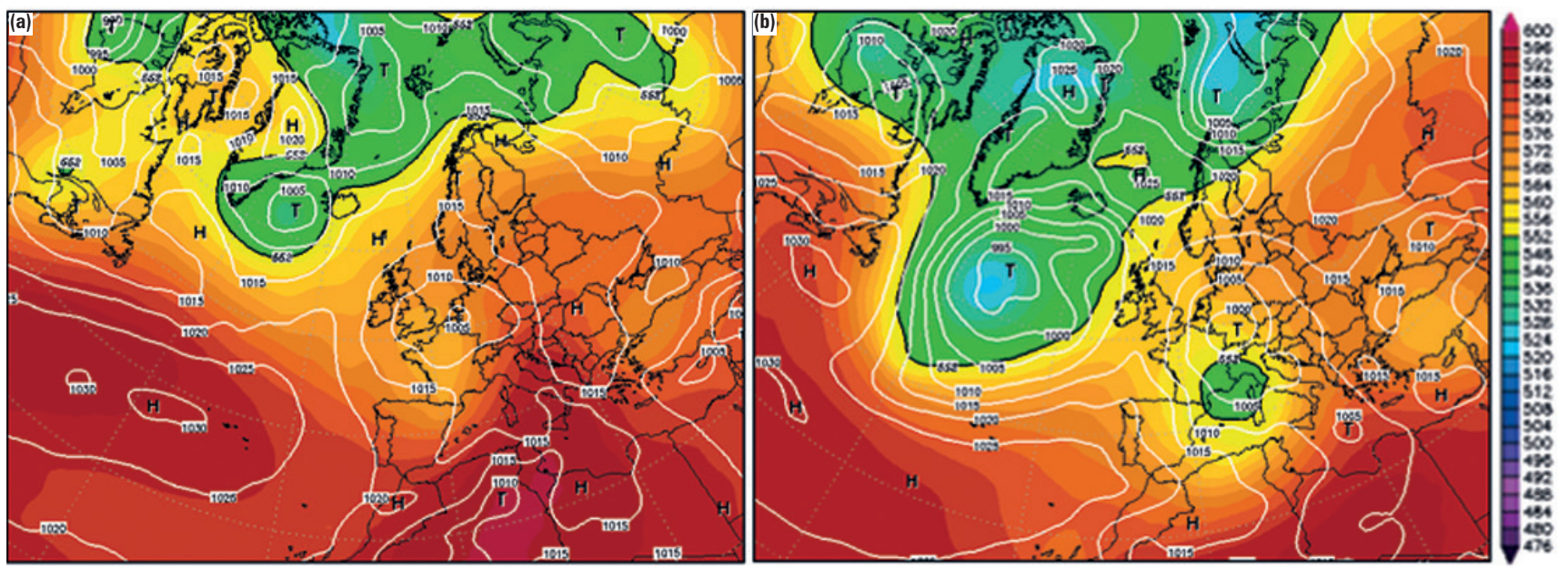

Figure 10. Analyse sur l'Europe et l'Atlantique Nord de la situation : (a) du 7 juillet 2001, (b) du 21 mai 2012. Source : www.wetterzentrale.de 


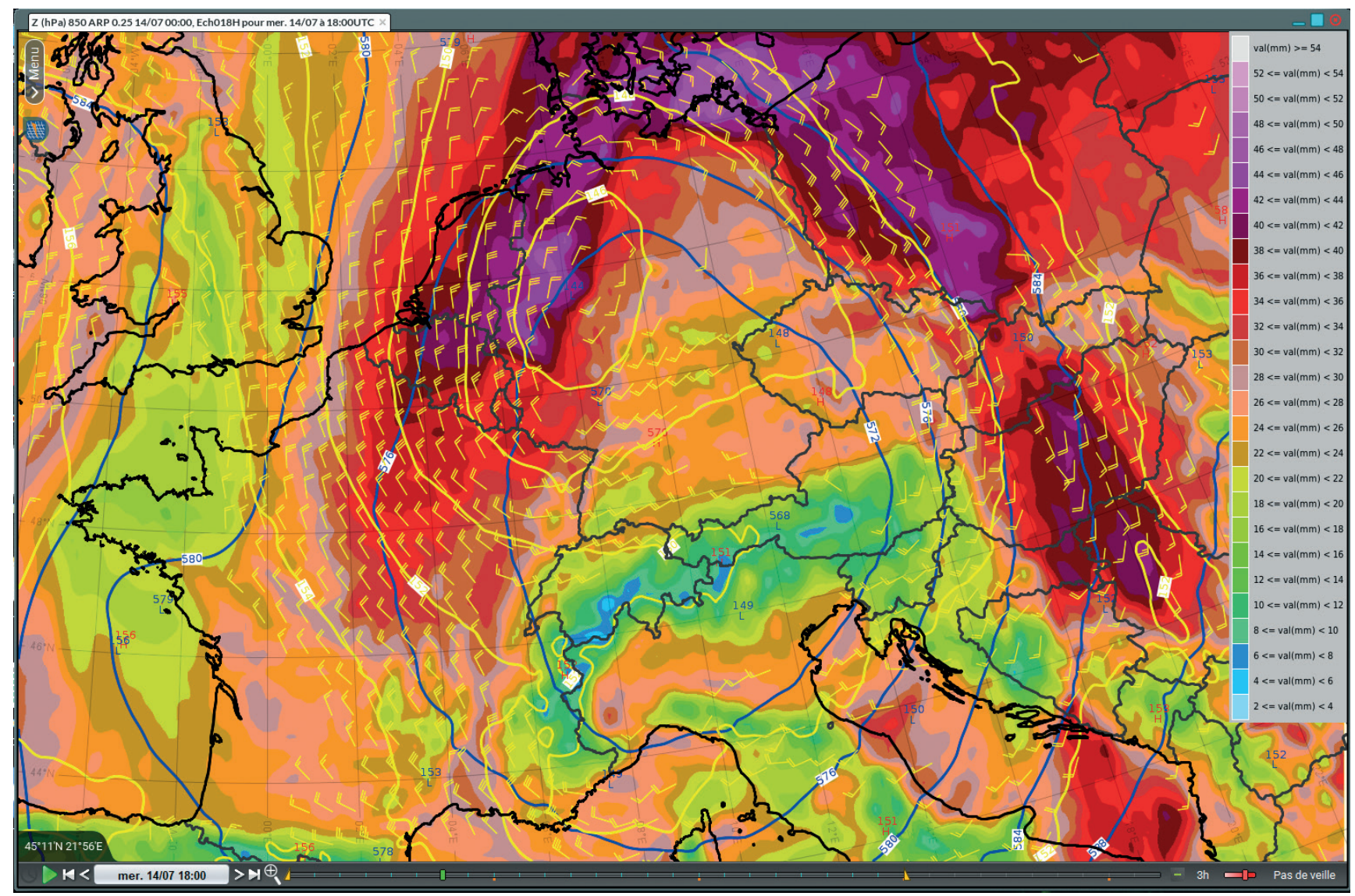

Figure 11. Géopotentiel à $500 \mathrm{hPa}$ (isolignes bleues), Pmer (isolignes jaunes), vent au niveau $850 \mathrm{hPa}$ (symboles) et eau précipitable analysés par Arpège le 14 juillet 2021 à 18 h TU. Source : Météo-France.

Ces inondations se produisent à la suite d'une succession de situations météorologiques favorables. Déjà, les mois de mai et juin sont particulièrement arrosés en Allemagne et sur les pays limitrophes, surtout ceux situés

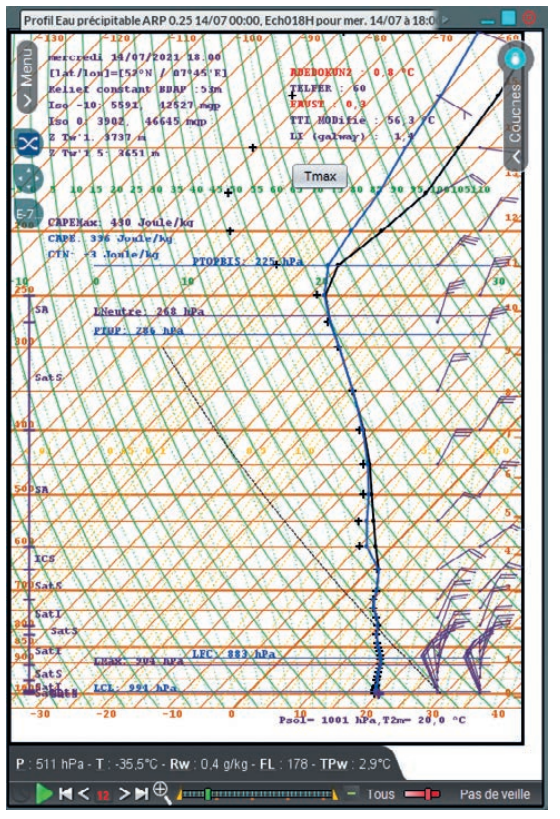

Figure 12. Profil vertical réalisé sur le flanc ouest de la goutte froide, là où les cumuls de précipitations sont les plus intenses, pour le 14 juillet 2021 à $18 \mathrm{~h}$ TU, base Arpège de $0 \mathrm{~h}$ TU. Source : Météo-France. en amont du bassin-versant complet du Rhin (figure 8). Le mois de juillet, en incluant ces précipitations, ne déroge pas. À grande échelle, les excédents sont souvent entre +20 et $+40 \%$ pour chacun de ces mois, avec des excédents nettement plus marqués localement.

Mais dans un contexte où les sols sont déjà humides, c'est un événement météorologique particulier qui est à l'origine de ces inondations. Il s'agit d'une goutte froide. Ce terme est employé pour décrire une dépression d'altitude (on parle de bas géopotentiel) qui s'isole du courant perturbé circulant vers des latitudes plus hautes. On peut également parler de cut-off. D'un point de vue physique, un bas géopotentiel est associé à de l'air froid d'altitude. Ici, la goutte froide est étendue et marquée, bloquée sur l'arc alpin entre une dorsale sur les îles Britanniques et une autre sur l'Europe de l'Est (figure 9). Elle est caractérisée par un bas géopotentiel de 568 damgp et une température inférieure à $-16^{\circ} \mathrm{C}$ à $500 \mathrm{hPa}$.

En saison estivale, cet air froid d'altitude entraîne un temps souvent instable. Bloquée entre des valeurs de géopotentiel plus élevées, une goutte froide engendre des précipitations notables sur plusieurs jours. En particulier, ses quadrants nord à ouest sont à surveiller attentivement au regard de cet enjeu. En effet, si la goutte froide est suffisamment étendue, elle va réussir à advecter de l'air chaud des latitudes plus au sud qui va venir la contourner jusqu'à son flanc ouest. Ce type de configuration est à l'origine de précipitations historiques exceptionnelles en France (figure 10) :

- nuit du 6 au 7 juillet $2001^{6}: 110 \mathrm{~mm}$ relevés en 24 heures à la station de ParisMontsouris, dont $85,0 \mathrm{~mm}$ en 6 heures, $50,2 \mathrm{~mm}$ en 2 heures et $33,3 \mathrm{~mm}$ en 1 heure, ou encore $121 \mathrm{~mm}$ à Bagneux ;

- 21 mai $2012^{7}$ : la station de Nancy enregistre $25,6 \mathrm{~mm}$ en 15 minutes, $43,0 \mathrm{~mm}$ en 30 minutes, $49 \mathrm{~mm}$ en 1 heure et 94,9 mm en 3 heures;

- fin mai $2016^{8}$ : inondations remarquables au nord de la Loire avec plus de $80 \mathrm{~mm}$ relevés en 4 jours sur ParisMontsouris et $130 \mathrm{~mm}$ sur Orléans.

6. http://pluiesextremes.meteo.fr/francemetropole/Orage-sur-Paris-et-la-PetiteCouronne.html

7. http://pluiesextremes.meteo.fr/francemetropole/Orage-violent-sur-Nancy.html 8. http://pluiesextremes.meteo.fr/francemetropole/Inondations-sur-Paris-et-en-regionCentre.html 

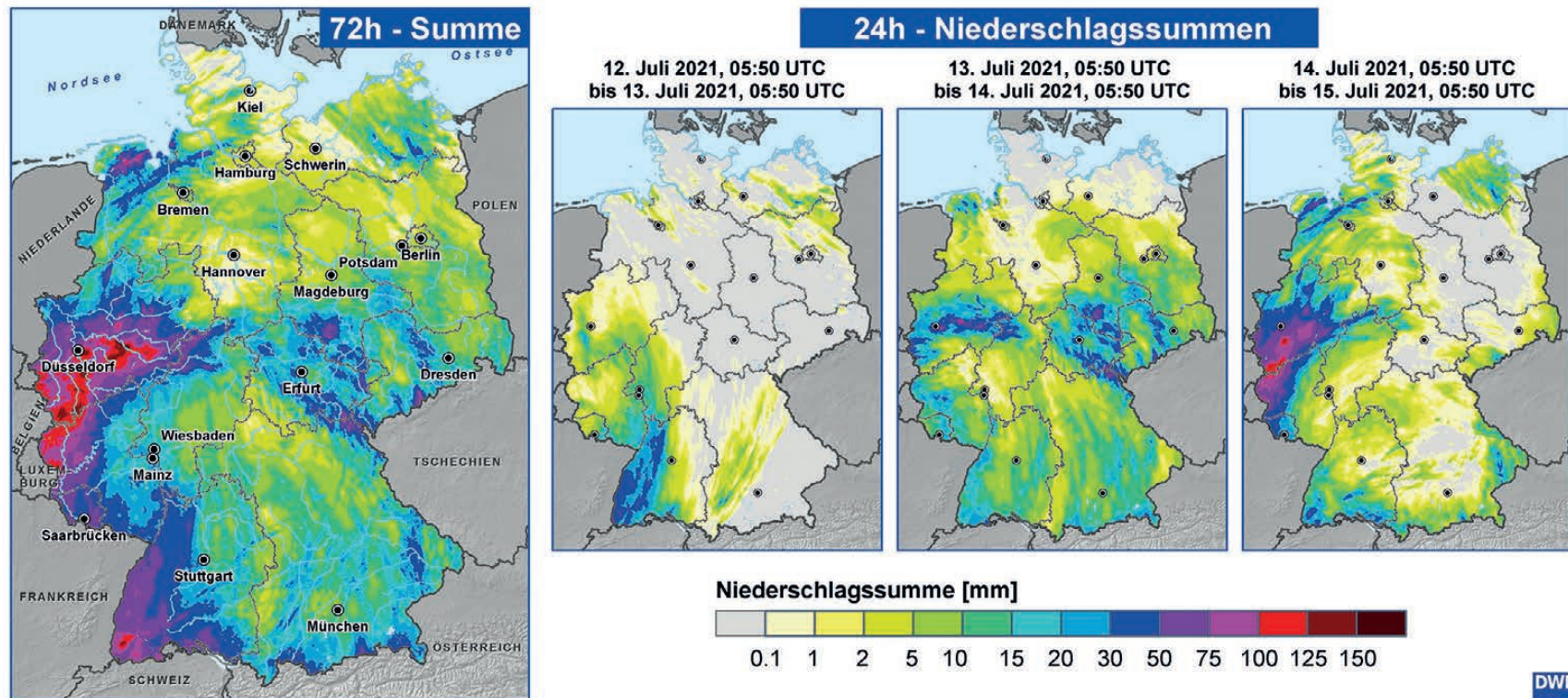

Niederschlagssumme [mm]

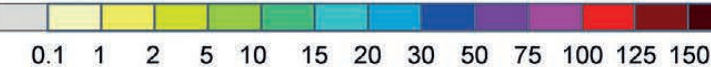

Klimadaten und Darstellung: ๑ Deutscher Wetterdienst 2021 (Stand: 15.07.2021); Geodaten: (๑) GeoBasis-DE/BKG 2020 (Stand: 01.01.2020).

Deutscher Wetterdienst Wetter und Klima aus einer Hand

Figure 13. Lames d'eau radar quotidiennes (à droite) et cumulées sur trois jours (à gauche) du 12 au 14 juillet 2021 en Allemagne. Source : DWD.

Pour la situation de l'été 2021, les valeurs d'eau précipitable s'enroulent autour du minimum (figure 11) et sont exceptionnelles pour la région, dépassant les $40 \mathrm{~mm}$. En raison d'un flux plus modéré en altitude et d'une dépression en surface sur ce secteur (décalage habituel des centres d'action entre surface et altitude), des précipitations pluvio-instables à caractère durable sont à redouter, souvent modulées par l'évolution diurne.
Les profils verticaux (figure 12) ne semblent pas élevés d'un point de vue thermique seul. Il faut alors regarder la température potentielle pseudo-adiabatique $T^{\prime}$ w ou la température du point de rosée $T_{\mathrm{d}}$, qui comparées à la température, donnent une indication de l'humidité relative. On voit alors un profil effectivement instable et saturé sur presque toute l'épaisseur en réponse à l'advection chaude et au long soulèvement synoptique autour de la goutte froide, confirmant le potentiel précipitant de la masse d'air.

Les cumuls quotidiens de précipitations marquent bien l'évolution $\mathrm{du}$ temps pluvio-instable autour de la goutte froide durant les trois jours (figure 13). D'abord sur la vallée du Rhin vers l'Alsace, les précipitations les plus fortes ceinturent ensuite une tranche centrale du pays,
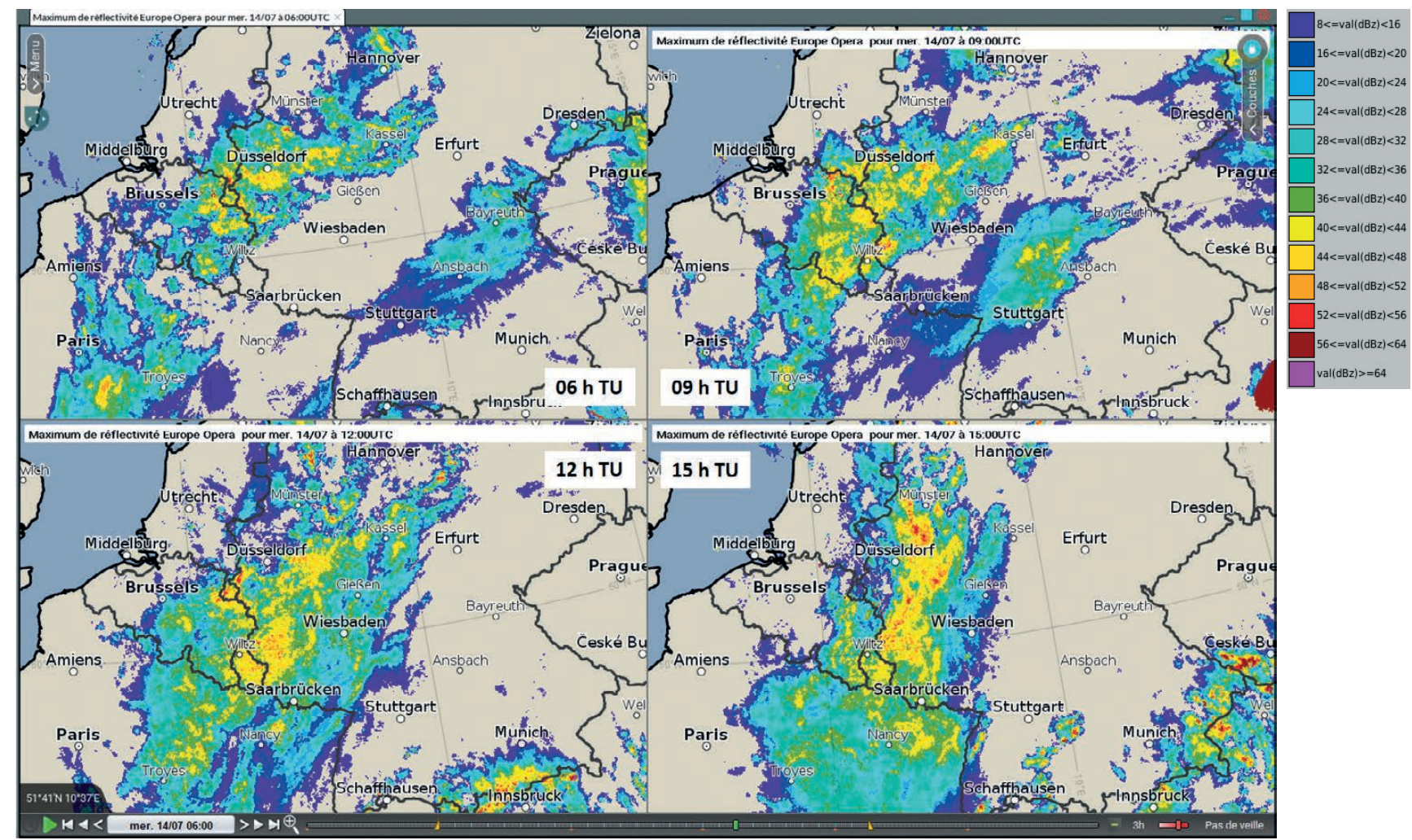

Figure 14. Suivi tri-horaire des réflectivités radar le 14 juillet de 6 h à 15 h TU. Source : Météo-France. 


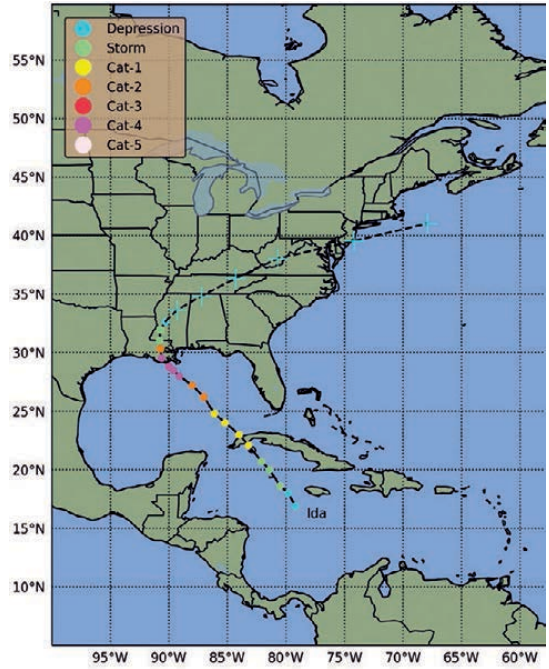

Figure 15. Trajectoire suivie par lda et intensité associée. Source : Plymouth State Weather Center.

avant de redescendre vers le sud-ouest. La faible mobilité de ces pluies instables vient accentuer les cumuls de précipitations sur la période, de même que la présence probable d'orages avec des quantités localement plus élevées.

Enfin, en suivant la journée du 14 juillet à l'aide des images radar (figure 14), le retour pluvio-instable est étendu et très actif. Il ne se décale que très lentement vers le sud-ouest en suivant l'enroulement cyclonique des vents autour de la goutte froide. Puis, à 12 et $15 \mathrm{~h}$ TU, des réflectivités plus intenses (couleur rouge) sont noyées dans la masse. Il s'agit d'orages apparaissant au cœur de ce retour pluvieux par (petite) évolution diurne.

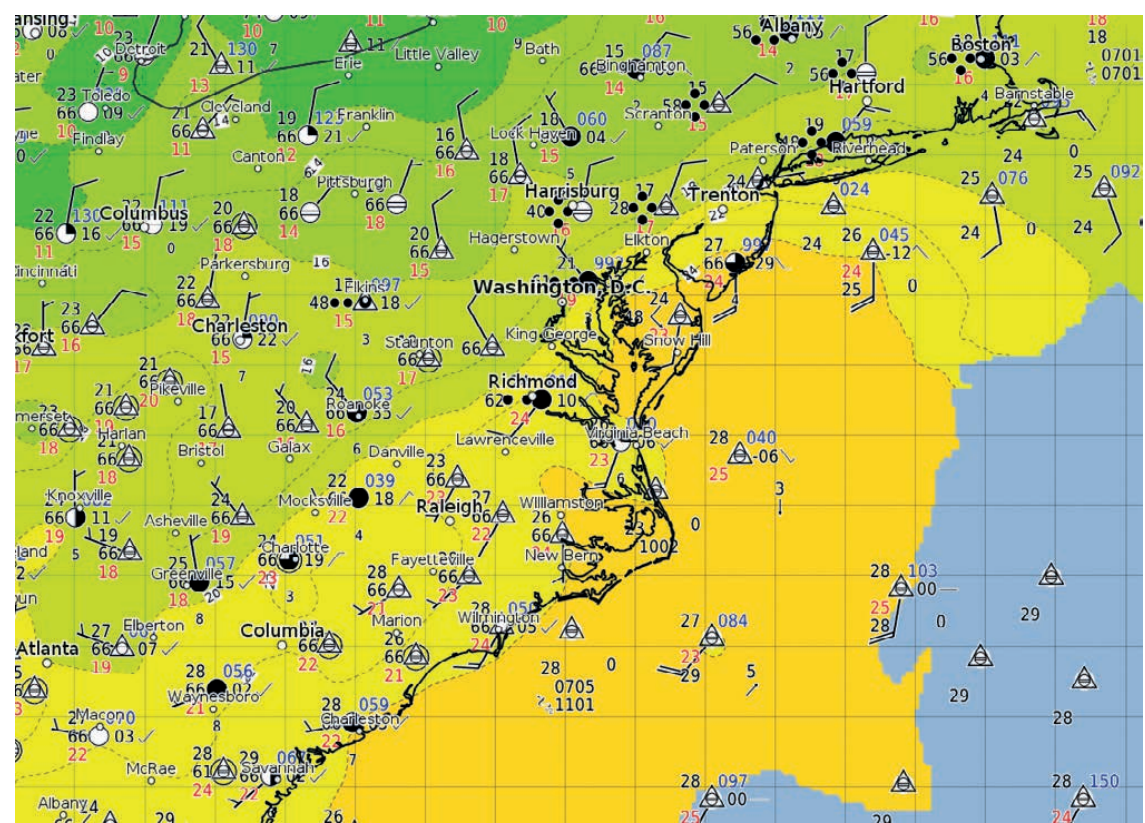

Figure 17. Données de stations météorologiques dans une partie de l'est des États-Unis, incluant les températures du point de rosée le 2 septembre 2021 à 0 h TU. Source : Météo-France.

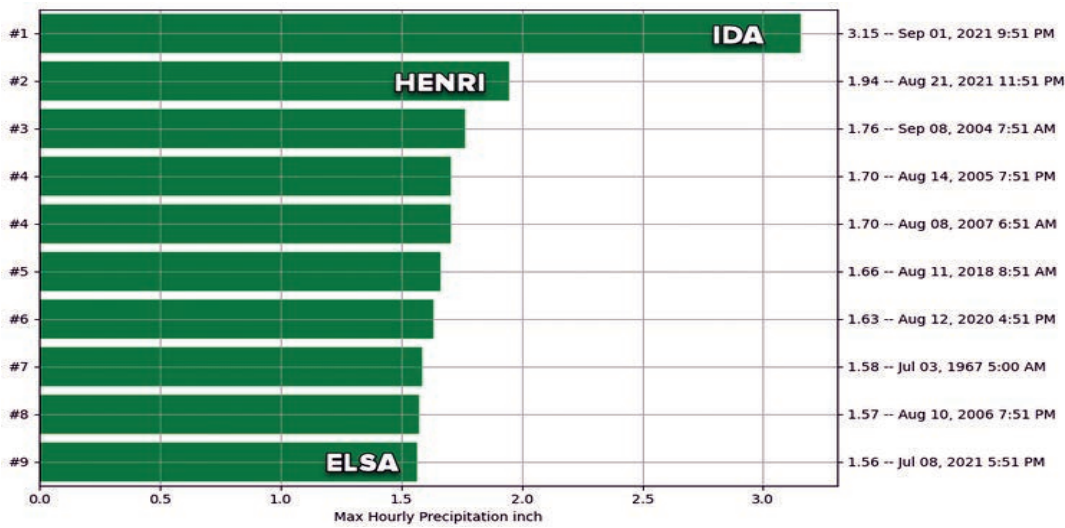

Figure 18. Records de pluies horaires à New York, station de Central Park. Source : Source : Noaa/ NWS

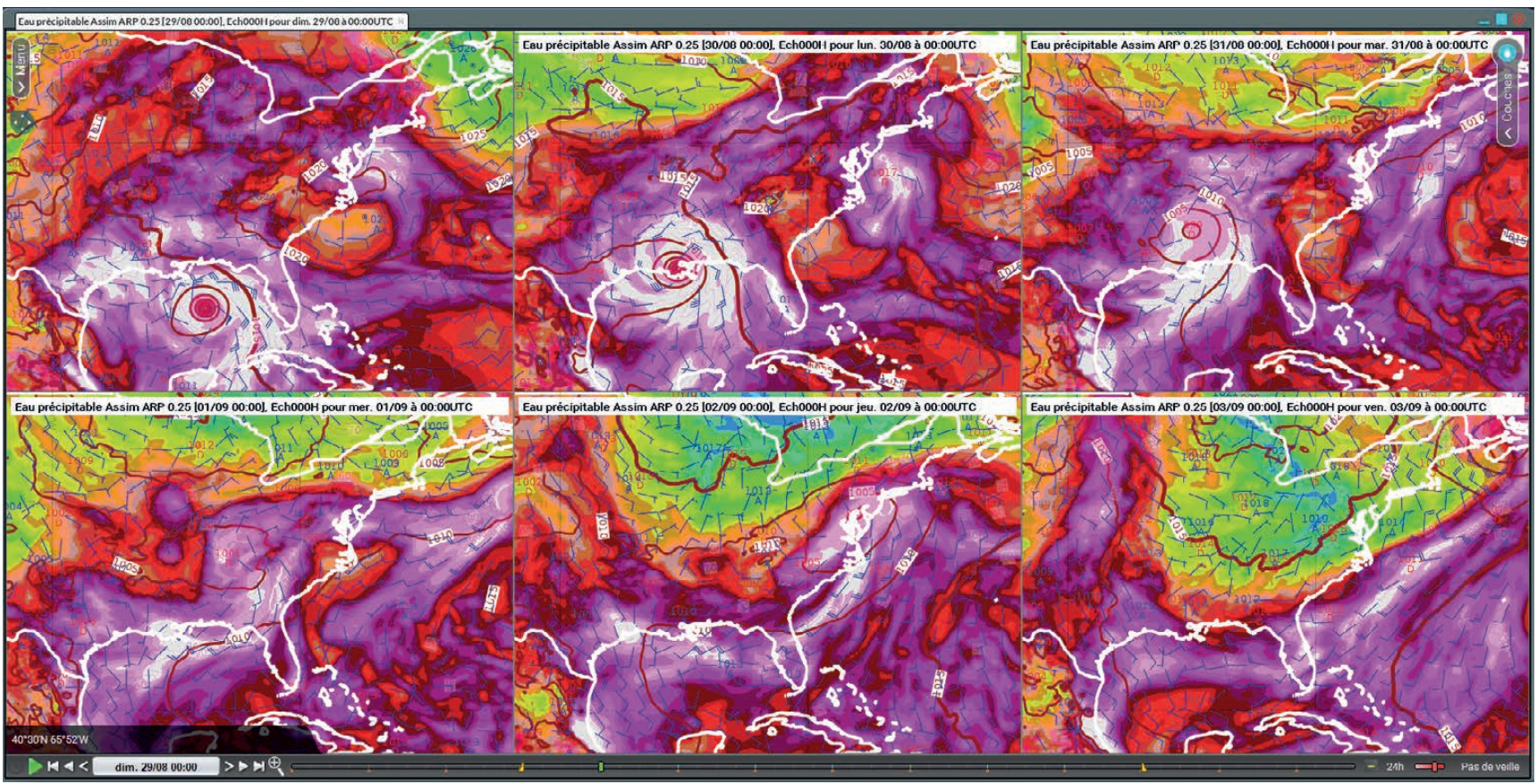

Figure 16. Pmer (isolignes marrons), vent à 10 mètres (symboles) et eau précipitable (couleurs, échelle proposée en figure 11). Analyses Arpège du 29 août au 3 septembre 2021. Source : Météo-France. 


\section{Ida : pluies record sur New York}

Dans les phénomènes précipitants extrêmes de l'été, le cyclone Ida tient une place à part. Ce système tropical se forme dans les Caraïbes, à proximité de la Jamaïque. Le stade de tempête tropicale est atteint le 26 août (figure 15). Ida

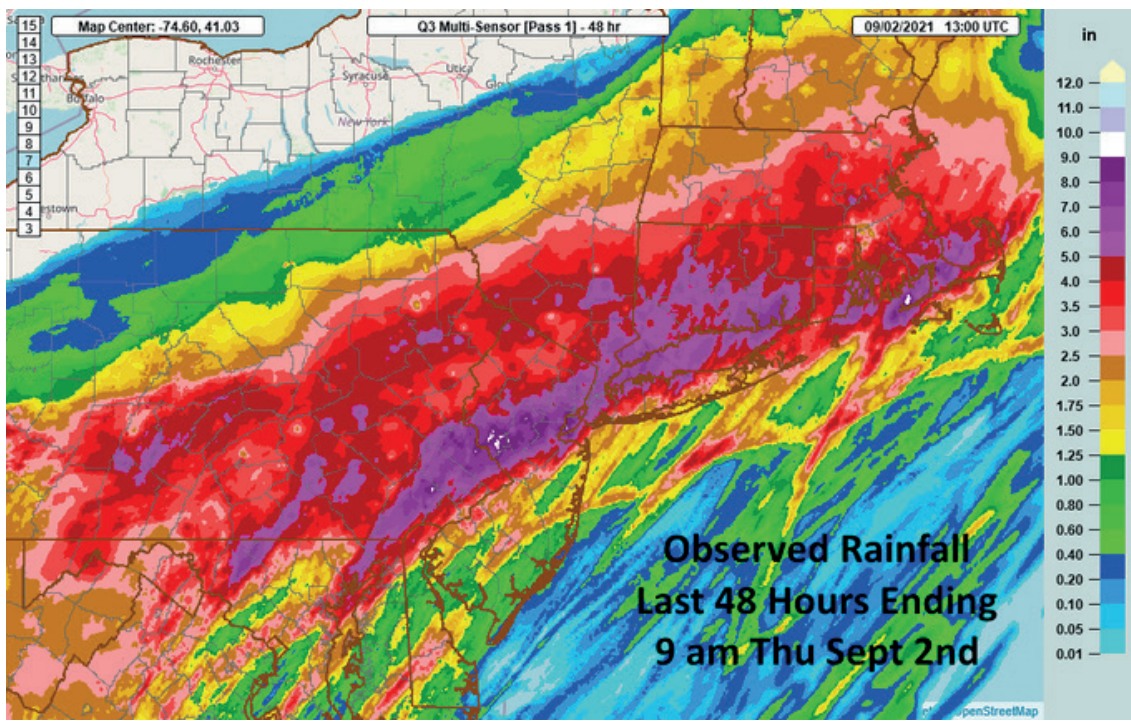

Figure 19. Cumuls de pluie observés en 48 heures au passage des restes d'lda autour de New York. L'échelle de couleur des précipitations est en pouces (1 pouce $=25,4 \mathrm{~mm}$ ). Source : Noaa/NWS. remonte vers le nord-nord-ouest en se renforçant. C'est en traversant le golfe du Mexique que son creusement devient brutal en atteignant la catégorie 4 et une valeur au centre estimée à $929 \mathrm{hPa}$ juste avant de toucher terre en Louisiane. Ida perd ses caractéristiques de système tropical au fur et à mesure de sa progression dans les terres en remontant le Mississippi.

\section{Bibliographie}

Mansouri Daneshvar M.R., Freund F., 2019. Examination of a relationship between atmospheric blocking and seismic events in the Middle East using a new seismo-climatic index. Swiss Journal of Geosciences, 112. doi: 10.1007/s00015-019-00343-4

Renaut D., 2020. 54, $4^{\circ} \mathrm{C}$ : la vallée de la Mort mérite bien son nom. La Météorologie, 111. doi:10.37053/lameteorologie-2020-0082

Stein A.F., Draxler R.R., Rolph G.D., Stunder B.J.B., Cohen M.D., Ngan F., 2015. NOAA's HYSPLIT atmospheric transport and dispersion modeling system. Bull. Amer. Meteor. Soc., 96, 2059-2077. doi: 10.1175/BAMS-D-14-00110.1 